\title{
Wann gibt's noch Teststreifen „auf Kasse“?
}

\begin{abstract}
Am 17. März hatte der Gemeinsamen Bundesausschuss (G-BA) beschlossen, dass Blut- und Urinzuckermessstreifen für nicht insulinpflichtige Typ-2-Diabetiker nur noch zulasten der GKV verordnet werden dürfen, wenn die Selbsmessung „,wirkliche Vorteile“ hat. Der Beschluss basiert auf Untersuchungen des IQWiG, das der Blutzuckerselbstmessung (BZSM) dieser Patienten den Nutzen abspricht, da sie keine direkten Konsequenzen auf die Therapie hätte. Vonseiten der Ärzteschaft hagelt es schon länger Kritik an dieser Sichtweise. Bis der Beschluss rechtskräftig ist, was frühestens am 1. Oktober der Fall sein kann, wird nun diskutiert und auch ein bisschen gerätselt, welche Situationen eine Basis für eine Teststreifenverordnung „, auf Kasse“ sein können.
\end{abstract}

\section{Das sagt der G-BA dazu}

\section{Missbrauch stoppen}

- Wenn Ärzte einen guten Grund für die Verordnung von Blutzuckerteststreifen bei nicht insulinpflichtigen Typ-2-Diabetikern finden, dann können sie 50 Messstreifen per Kassenrezept verschreiben. Die Einschätzung der Situation liegt im Ermessen des Arztes, und eine Behandlungssituation ist nicht strikt zeitlich begrenzt.

Beispiele für eine Verordnungssituation sind eine Influenza mit erhöhter Wahrscheinlichkeit für eine Stoffwechselentgleisung oder die Einstellung auf ein neues Antidiabetikum mit erhöhter Hypoglykämiegefahr. Es muss aber immer eine Einzelfallentscheidung sein, die plausibel ist. So solle verhindert werden, dass nicht insulinpflichtige Patienten immer wieder messen, ohne dass das Verhalten oder die Therapie geändert werden oder dass sie Teststreifen zu Hause horten, die sie nie aufbrauchen können. Diese Form von Missbrauch sei vorgekommen. Stabil eingestellte Patienten mit oraler Therapie würden ohnehin regelmäßig beim Arzt kontrolliert. Hier habe die Selbstmessung keinen Nutzen. Man hofft, so von den jährlich in Deutschland etwa 900 Mio. Euro für Diabetestests mehrere 100 Mio. einsparen zu können.

\section{Das sagen diabetesDE und DDG dazu}

\section{Kostenverlagerung zum Arzt}

- Es sei nicht hinnehmbar, dass finanzielle Aspekte vor der Patientensicherheit rangierten, äußerte sich Prof. Thomas Danne, Vorstandsvorsitzender von diabetesDE und Präsident der Deutschen Diabetes-Gesellschaft (DDG) in einer aktuellen Stellungnahme.

Man halte das zugrundeliegende IQWiG-Gutachten, das keinen therapeutischen Nutzen der regelmäßigen BZSM erkennen konnte, für unzureichend. Zudem sei die BZSM ein unverzichtbarer Bestandteil einer strukturierten Diabetesschulung und damit einer modernen Therapie bei Diabetes, denn sie stärke den selbstverantwortlichen Umgang der Patienten mit ihrer Erkrankung. Der G-BA nehme den Patienten mit diesem Entscheid das einzige Mittel der persönlichen Schadensbegrenzung im Fall einer drohenden oder eingetretenen Hypoglykämie aus der Hand. Auch sei eine instabile Stoffwechsellage durch die Ermittlung der $\mathrm{HbA}_{1 c^{-}}$ Werte in den Praxen gar nicht feststellbar. Bislang sei daher auch ungeklärt, ob die neue Regelung das Gebot der Wirtschaftlichkeit erfüllen könne oder ob nicht Mehrkosten durch vermehrte Messungen aktueller Blutzuckerwerte in der ambulanten vertragsärztlichen Versorgung entstünden.

Das sagt eine niedergelassene Diabetologin dazu

\section{In vielen Situationen kann nur der Patient selbst kontrollieren}

- Blutzuckerselbstmessungen sind bei nicht insulinpflichtigen Typ-2-Diabetikern unentbehrlich, wenn Patienten mit einer oralen Antidiabetikatherapie über mögliche Hypoglykämien berichten, so Dr. Veronika Hollenrieder aus München, Vorstandsmitglied beim Berufsverband der niedergelassenen Diabetologen in Bayern. Hypoglykämien könnten nur durch sofortige Messungen verifiziert werden, und sie seien für die Patienten ein großes Angstthema. „Die Selbstmessung ist hier unverzichtbar, um Vertrauen zu Medikament und Arzt aufbauen zu können“, so Hollenrieder. Desweiteren hält sie sporadische Messungen für Patienten, die sportlich sehr aktiv sind, für wichtig, und drittens für Patienten, denen man starke postprandiale Blutzuckerspitzen visualisieren muss - sozusagen als pädagogisches Hilfsmittel. „In diesen Situationen kann die Kontrolle nur durch eine Selbstmessung erfolgen." Sie empfiehlt für Patienten mit oraler Antidiabetikatherapie als „Minimalkontrolle“ ein Blutzuckertagesprofil pro Monat, das sind jeweils sieben Teststreifen - somit im Quartal 21 Teststreifen. Mit einer Dose á 50 Streifen würde der Patient also ein halbes Jahr auskommen. Bei einem Durchschnittspreis von 25 bis 35 Euro für 50 Streifen wären wir dann im Jahr bei 50 bis 70 Euro Kosten für einen Patienten. 